PROCEEDINGS OF THE AMERICAN MATHEMATICAL SOCIETY

Volume 124, Number 9, September 1996

\title{
ON THE SPECTRAL CHARACTER OF TOEPLITZ OPERATORS ON PLANAR REGIONS
}

\author{
GHOLAMREZA AKBARI ESTAHBANATI
}

(Communicated by Albert Baernstein II)

\begin{abstract}
Self-adjoint Toeplitz operators on multiply connected planar regions are investigated using theta functions on the double. An explicit resolvent form for self-adjoint Toeplitz operators on a Hardy space associated with any representing measure on a $g$-holed planar region is given via reproducing kernels in terms of theta functions on $\mathbb{C}^{g}$. This resolvent formula is a generalization of an analogous formula obtained by K. F. Clancey (1991) for the case of harmonic measure. Applications of this resolvent form to the spectral type of the self-adjoint Toeplitz operators are described.
\end{abstract}

\section{INTRODUCTION}

The resolvent formula and spectral analysis of the self-adjoint Toeplitz operators on the Hardy space $H^{2}(d m)$ on the unit disc have been worked out in detail by M. Rosenblum [10]. Here $d m$ refers to Lebesgue measure on the unit circle. For an arbitrary real $\phi$ in $L^{\infty}(d m)$, Rosenblum has "concretely" diagonalized the selfadjoint Toeplitz operator $T_{\phi}$ on the unit circle. Specifically, it is shown that $T_{\phi}$ is absolutely continuous unless $\phi \equiv$ constant. J. D. Pincus and J. Xia [9] have also studied the self-adjoint case when the region $D$ is multiply connected planar having $g$ holes, $g \geq 1$, and $m_{a}$ is harmonic measure for $D$ relative to a fixed point $a \in D$. They show that in the self-adjoint case the absolutely continuous spectral theory of $T_{\phi}$ is related to the absolutely continuous spectral theory of $T_{\phi_{i}}, \phi_{i}=\left.\phi\right|_{b_{i}}$, on Hardy spaces of analytic functions on the simply connected regions bounded by $b_{i}$, $i=0,1, \ldots, g$, and $\bigcup_{i=0}^{g} b_{i}=\partial D$. More specifically, if $\phi \in L^{\infty}\left(d m_{a}\right)$ and $\bar{\phi}=\phi$ is not constant on $b_{i}$, then one gets an interval $\left[m_{i}, M_{i}\right]$ of absolutely continuous spectrum, where $m_{i}=\operatorname{ess} \inf \left(\phi_{i}\right), M_{i}=\operatorname{ess} \sup \left(\phi_{i}\right), i=0,1, \ldots, g$. K. F. Clancey [5] investigated self-adjoint Toeplitz operators on least harmonic majorant Hardy spaces by developing a generalization of M. Rosenblum's resolvent formula. His investigation is based upon a resolvent formula for higher genus. He considers the region $D$ as a regular region on its double $X$. When viewed in this manner, it is possible to describe the orthogonal complement of $H^{2}$ using meromorphic functions on $X$. In this analysis, Riemann theta functions enter naturally into the resolvent formula. The techniques rely heavily on results from Riemann surfaces

Received by the editors September 13, 1994 and, in revised form, January 30, 1995 and March 3, 1995.

1991 Mathematics Subject Classification. Primary 47B35.

Key words and phrases. Riemann surface, double, representing measure, theta function, Hardy space, Toeplitz operator.

(C)1996 American Mathematical Society 
and theta functions. Clancey shows that when the symbol $\phi \in L^{\infty}\left(d m_{a}\right)$ is a simple or an analytic function on the boundary of the region, then the singular continuous component of the spectral measure of $T_{\phi}$ is trivial. Moreover, if one restricts attention to doubly connected planar regions, then this property holds for any real symbol $\phi$ in $L^{\infty}\left(d m_{a}\right)$. His conjecture in this setting is that in general the singular continuous component is trivial even when one considers not only harmonic measures but an arbitrary non-negative representing measure.

In the case of arbitrary representing measures, when the symbols $\phi$ are those considered by Clancey, the Toeplitz operator $T_{\phi}$ is unitarily equivalent to a finite dimensional perturbation of a Toeplitz operator with the same symbol acting on the least harmonic majorant Hardy space $H^{2}\left(d m_{a}\right)$. Therefore, assuming the results in Clancey [5], one may apply the abstract perturbation theory to study the spectral character of $T_{\phi}$. Let us point out here that, in general, a finite dimensional perturbation of an operator may cause a significant effect on the spectral behavior of that operator (see example 1 of W. F. Donoghue [6]).

The main goal of this paper is to establish a generalization of Clancey's resolvent formula for self-adjoint Toeplitz operators on Hardy spaces associated with any non-negative representing measure. Using this as a tool, it is concluded that on an arbitrary multiply connected planar region, a self-adjoint Toeplitz operator whose symbol is either analytic or a simple function cannot have a singular continuous component. Further, when the region is doubly connected, an arbitrary self-adjoint Toeplitz operator does not have a singular continuous component.

This paper is organized as follows. In Section 1 we will recall some preliminaries concerning notation and definitions of Hardy spaces and theta functions associated with doubles of multiply connected planar regions. Moreover, in this section we will describe an explicit formula for the reproducing kernel for Hardy spaces $H_{\mathcal{D}}^{2}(d m)$ on multiply connected planar regions associated with representing measures, in terms of their related theta functions. In Section 2 we will generalize Clancey's resolvent formula [5]. In Theorem 1, we will give an explicit resolvent formula for the selfadjoint Toeplitz operators $T_{\phi}$ on Hardy spaces $H_{\mathcal{D}}^{2}(d m)$. This was made possible by the explicit description of the reproducing kernels obtained by J. A. Ball and K. F. Clancey [2]. We will give the proof of Theorem 1 in this section also. In Section 3 , we will present the corollaries for Theorem 1 about the spectral characters of $T_{\phi}$ on these Hardy spaces.

\section{Preliminaries}

Let $D$ be a bounded planar region having $g$ holes, $b_{0}$ the boundary of the unbounded component of the complement of $D$, and $b_{1}, \ldots, b_{g}$ the boundaries of the $g$ holes. The boundary $\partial D$ of $D$ which consists of $b_{0}, \ldots, b_{g}$ is assumed to be analytic and oriented positively. Let $X$ be the double of $\bar{D}$ with the usual complex structure determined by requiring the mapping $J: X \rightarrow X$, interchanging points in $D$ with their twins, to be an anti-conformal involution on $X$. The double $X$ is a compact Riemann surface of genus $g$. We mark $X$ by the symmetric canonical homology basis $a_{1}, \ldots, a_{g}: b_{1}, \ldots, b_{g}$ for $H_{1}(X)=H_{1}(X, \mathbb{Z})$, where $a_{i}=\alpha_{i}-J \alpha_{i}$, and $\alpha_{i}$ is a cross cut from a fixed point $p_{0}$ on $b_{0}$ to a point on $b_{i}, i=1, \ldots, g$. Let $d w_{1}, \ldots, d w_{g}$ be the basis for $\Omega(X)$, the set of holomorphic functions on $X$, dual to the above symmetric canonical homology basis. The B-period matrix $\tau$ has the form $\tau=\left[\int_{b_{j}} d w_{i}\right]=i P$, where $P$ is a symmetric positive definite $g \times g$ matrix. 
Let $m$ be a representing measure in the set $M_{a}(X)$ of non-negative representing measures based at a fixed point $a$ in $D$ with the critical divisor $\mathcal{D}_{m}$. Let $V_{a}$ denote the collection of all divisors $\mathcal{D}$ in the $g$-fold symmetric product $X^{(g)}$ which provides the representation $(\omega)=\mathcal{D}+J(\mathcal{D})-a-J a$ for some symmetric meromorphic differential $\omega$ in the set $M^{1}(X)$ of meromorphic differentials on $X$ with $\left.\omega\right|_{\partial D}$ in $M_{a}$. For a given divisor $\mathcal{D}$ in $V_{a}$, let $\mathcal{D}^{+}=\left.\mathcal{D}\right|_{\bar{D}}, \mathcal{D}^{-}=\left.\mathcal{D}\right|_{J \bar{D}}$. The Hardy space associated with the representing measure $m, H_{\mathcal{D}}^{2}(d m)$, is the closure in $L^{2}(d m)$ of $L\left(\bar{D}: \mathcal{D}^{+}\right)$, where $L\left(\bar{D}: \mathcal{D}^{+}\right)$is the subspace of the complex vector space $M(\bar{D})$ of meromorphic functions $f$ on $\bar{D}$ having the divisors $(f) \geq-\mathcal{D}^{+}$. Similarly, $K_{\mathcal{D}}^{2}(d m)$ denotes the closure in $L^{2}(d m)$ of functions $f$ meromorphic on $J \bar{D}$ satisfying $(f) \geq-\mathcal{D}^{-}$. Also, we let $K_{\mathcal{D}}^{2, a}(d m)$ denote the closure in $L^{2}(d m)$ of functions $f$ meromorphic on $J \bar{D}$ satisfying $(f) \geq-\mathcal{D}^{-}+J a$. Notice that if $\mathcal{D}=\mathcal{D}_{m_{a}}$, then $H_{\mathcal{D}_{m_{a}}}^{2}\left(d m_{a}\right)$ is the familiar least harmonic majorant Hardy space $H^{2}\left(d m_{a}\right)$. Moreover, if $\mathcal{D}^{+}$is zero, then $H_{\mathcal{D}}^{2}(d m)$ is the closure in $L^{2}(d m)$ of functions holomorphic on a neighborhood of $\bar{D}$. In the case where $\mathcal{D}^{+}$is non-zero the space $H_{\mathcal{D}}^{2}(d m)$ is a Hardy space with singularities. This is because the elements in $L\left(\bar{D}: \mathcal{D}^{+}\right)$can have poles at the points in $\mathcal{D}^{+}$. One has the following orthogonal decomposition of $L^{2}(d m)$ :

$$
L^{2}(d m)=H_{\mathcal{D}}^{2}(d m) \oplus K_{\mathcal{D}}^{2, a}(d m) .
$$

The proof of this fact is in K. F. Clancey [4].

The Abel-Jacobi map $\Phi_{0}$ based at a fixed point $p_{0}$ on $b_{0}$ is defined on $X$ by

$$
\Phi_{0}(p)=\int_{p_{0}}^{p} d \vec{w} \bmod \left(\mathbb{Z}^{g}+\tau \mathbb{Z}^{g}\right)
$$

and the Riemann theta function associated with $\tau$ is defined on $\mathbb{C}^{g}$ by

$$
\theta(\underline{z})=\theta(\underline{z}, \tau)=\sum_{\underline{n} \in \mathbb{Z}^{g}} \exp \left\{2 \pi i\left(\frac{1}{2} \underline{n}^{t} \tau \underline{n}+\underline{n}^{t} \underline{z}\right)\right\}
$$

The theta function is an entire and quasi-periodic in the sense that for $\underline{z} \in \mathbb{C}^{g}$ and $\underline{m}, \underline{n}$ in $\mathbb{Z}^{g}$,

$$
\theta\left(\underline{z}+\underline{m}+\underline{n}^{t} \tau\right)=\exp \left\{2 \pi i\left(-\frac{1}{2} \underline{n}^{t} \tau \underline{n}-\underline{n}^{t} \underline{z}\right)\right\} \theta(\underline{z}) .
$$

In particular, $\theta$ is $\mathbb{Z}^{g}$ periodic. Since the B-period matrix of the marked double $X$ has the form $\tau=i P$, the antiholomorphic involution map $J$ on the Jacobian variety $\operatorname{Jac}(X)=\mathbb{C}^{g} /\left(\mathbb{Z}^{g}+\tau \mathbb{Z}^{g}\right)$ defined by $J([\underline{z}])=-[\underline{\bar{z}}]$ is a well-defined map. We remark that the theta function associated with the B-period matrix $\tau$ of a double of genus $g$ never vanishes in $\mathbb{R}^{g}$. This result is in Fay [7].

Let $e_{*}=\frac{1}{2}\left(\mu_{*}+\tau \lambda_{*}\right), \mu_{*}, \lambda_{*} \in \mathbb{Z}^{g} / 2 \mathbb{Z}^{g}$, be a fixed nonsingular odd-half period. This means that the complex gradiant $\nabla \theta\left(e_{*}\right) \neq 0$. The existence of $e_{*}$ is established in D. Mumford [8]. We denote the theta function with characteristics $\underline{a}=\frac{1}{2} \lambda_{*}$ and $\underline{b}=\frac{1}{2} \mu_{*}$ by $\theta_{*}$. Thus,

$$
\theta_{*}(\underline{z})=\theta_{*}(\underline{z}, \tau)=\theta\left[\underline{a}[\underline{b}](\underline{z}, \tau)=\exp \left\{2 \pi i\left(\frac{1}{2} \underline{a}^{t} \tau \underline{a}+\underline{a}^{t}(\underline{z}+\underline{b})\right)\right\} \theta(\underline{z}+\underline{b}+\tau \underline{a}) .\right.
$$

The Hardy spaces $H_{\mathcal{D}}^{2}(d m)$ associated with representing measures $m$ are reproducing kernel Hilbert spaces. This means that for a given $z$ in $D \backslash \operatorname{supp} \mathcal{D}$, there is an element $k_{z}^{\mathcal{D}}$ in $H_{\mathcal{D}}^{2}(d m)$ satisfying $f(z)=\left\langle f, k_{z}^{\mathcal{D}}\right\rangle$, for any $f$ in $H_{\mathcal{D}}^{2}(d m)$, where $\langle\cdot, \cdot\rangle$ denotes the inner product in $H_{\mathcal{D}}^{2}(d m)$. Here we describe an explicit formula of J. A. Ball and K. F. Clancey for the reproducing kernels $k_{z}^{\mathcal{D}}$ of the Hardy spaces $H_{\mathcal{D}}^{2}(d m)$ : 
let $\mathcal{D}$ be a given element in $V_{a}$ providing a representation $(\omega)=\mathcal{D}+J(\mathcal{D})-a-J a$ for an $\omega$ in $M^{1}(X)$ with $d m=\left.\omega\right|_{\partial D}$. Also, let $X_{0}$ denote the image of $X$ in $\operatorname{Jac}(X)$ under the Abel-Jacobi map $\Phi_{0}$. Then, for a given $z$ in $D \backslash \operatorname{supp} \mathcal{D}$, the reproducing kernel $k_{z}^{\mathcal{D}}(\cdot)=k(\cdot, z: \mathcal{D})$ for the space $H_{\mathcal{D}}^{2}(d m)$ is given by

$$
\begin{aligned}
k_{z}^{\mathcal{D}}(\zeta) & =k(\zeta, z: \mathcal{D}) \\
& =\frac{\theta(\zeta+\bar{z}-a-\bar{a}-t) \theta(t) \theta_{*}(\zeta-J a) \theta_{*}(a-J z)}{\theta(\zeta-a-t) \theta(\bar{z}-\bar{a}-t) \theta_{*}(\zeta-J z) \theta_{*}(a-J a)}, \quad \zeta \in X_{0},
\end{aligned}
$$

where $t=\Phi_{0}(\mathcal{D})-\Phi_{0}(a)+\left[\Delta_{0}\right]$. The function $k_{z}^{\mathcal{D}}$ is a meromorphic function with the divisor of the form

$$
\left(k_{z}^{\mathcal{D}}\right)=\mathcal{D}_{z}-\mathcal{D}+J a-J z,
$$

where $\mathcal{D}_{z} \geq 0$ is of degree $g$. Note that $t$ depends only on $\mathcal{D}$.

\section{THEOREM 1}

Let $P$ denote the orthogonal projection of $L^{2}(d m)$ onto $H_{\mathcal{D}}^{2}(d m)$. Given $\phi$ in $L^{\infty}(d m)$ one defines the Toeplitz operator $T_{\phi}$ with the symbol $\phi$ as the operator on $H_{\mathcal{D}}^{2}(d m)$ given by

$$
T_{\phi} f=P(\phi f), \quad f \in H_{\mathcal{D}}^{2}(d m) .
$$

The Toeplitz operator $T_{\phi}$ is self-adjoint if and only if the symbol $\phi$ is real-valued.

One can associate with any given element $\mathcal{D} \in V_{a}$ the divisor $\mathcal{D}_{1}$ as follows. Let $z_{1}, z_{2}, \cdots, z_{k}$ be those elements in supp $\mathcal{D}$, repeated according to their orders, for which $J z_{i} \notin \operatorname{supp} \mathcal{D}, i=1, \ldots, k$. Let $\mathcal{D}_{1}$ be the divisor

$$
\mathcal{D}_{1}=z_{1}-J z_{1}+\cdots+z_{k}-J z_{k} .
$$

Evidently, $J \mathcal{D}+\mathcal{D}_{1}=\mathcal{D}$. The main theorem in this paper is

Theorem 1. Let $\phi$ be a real-valued element in $L^{\infty}(d m)$ and let $\mathcal{D} \in V_{a}$. Suppose $T_{\phi}$ is the associated self-adjoint Toeplitz operator on $H_{\mathcal{D}}^{2}(d m)$. For a given $z$ in $D \backslash \operatorname{supp} \mathcal{D}$ let $k_{z}^{\mathcal{D}}$ denote the reproducing kernel in $H_{\mathcal{D}}^{2}(d m)$. Then for any complex number $\lambda$ satisfying $\operatorname{Im} \lambda \neq 0$, we have the following:

$$
\begin{aligned}
\left\langle\left( T_{\phi}\right.\right. & \left.-\lambda)^{-1} k_{z}^{\mathcal{D}}, k_{z}^{\mathcal{D}}\right\rangle \\
& =C(z) \frac{\theta\left(\omega_{z}+t-\Phi_{0}\left(\mathcal{D}_{1}\right)-\frac{1}{2 \pi i} \int_{\partial D} \log (\phi-\lambda) d \vec{w}\right)}{\theta\left(t-\Phi_{0}\left(\mathcal{D}_{1}\right)-\frac{1}{2 \pi i} \int_{\partial D} \log (\phi-\lambda) d \vec{w}\right)} e^{-\int_{\partial D} \log (\phi-\lambda) d m_{z}},
\end{aligned}
$$

where $t$ is a constant, $\omega(z)$ is a constant depending only on $z$, and $C(z)$ is a constant depending only on $\mathcal{D}, z$.

This formula without the theta function factors is precisely the classical result in genus zero (see M. Rosenblum [10]). The case when $m=m_{a}$ is the harmonic measure based at $a$ and the Hardy space is the least harmonic majorant Hardy space $H^{2}\left(d m_{a}\right)$ appears in work of K. F. Clancey [5].

Proof. We first solve the Toeplitz equation $\left(T_{\phi}-\lambda\right) x=k_{z}^{\mathcal{D}}$ in $H_{\mathcal{D}}^{2}(d m)$ for $\operatorname{Im} \lambda \neq 0$. To do this, we try to solve an inhomogeneous Hilbert barrier problem with the symbol $G=(\phi-\lambda)$. Let $\log (\phi-\lambda)$ be the principal determination of the logarithm of the function $(\phi-\lambda)$, where $-\pi<\operatorname{ImLog} z \leq \pi$, also, let $\phi$ be smooth. Fix a 
point $b$ in $X \backslash \bar{D}$ and let $A(t, p) d t$ be the multiplicative Cauchy kernel based at $b$. Then for a fixed $\lambda \in \mathbf{C}$ with $\operatorname{Im} \lambda \neq 0$, the function $H$ defined on $X \backslash \partial D$ by

$$
H(p)=\exp \left(-\frac{1}{2 \pi i} \int_{\partial D} \log (\phi-\lambda) A(t, p) d t\right)
$$

is a multiple-valued holomorphic function belonging to the homology character $\chi$ with

$$
\chi\left(a_{j}\right)=1, \quad \chi\left(b_{j}\right)=\exp \left(-\int_{\partial D} \log (\phi-\lambda) d w_{j}\right), \quad j=1, \ldots, g .
$$

Moreover, $H$ has continuous extensions $H^{+}$(respectively, $H^{-}$) a.e. $m$ to $\partial D$ from inside (respectively, outside of $\bar{D}$ ) satisfying

$$
(\phi-\lambda) H^{+}=H^{-} \text {a.e. } m \text { on } \partial D .
$$

See Clancey [3] or E. I. Zverovich [11]. One can use theta functions to remove this multiplicative behavior of the function $H$ as follows. Let $t$ be a fixed element in $\Phi_{0}(\mathcal{D})-\Phi_{0}(a)+\left[\Delta_{0}\right]$, and for a fixed point $z$ in $D \backslash \operatorname{supp} \mathcal{D}$ define $f_{z}$ on $X$ by

$$
\begin{aligned}
f_{z}(p)= & \frac{\theta\left(\int_{p_{0}}^{p} d \vec{w}+\bar{z}+t-\Phi_{0}\left(\mathcal{D}_{1}\right)-\frac{1}{2 \pi i} \int_{\partial D} \log (\phi-\lambda) d \vec{w}\right)}{\theta\left(\int_{p_{0}}^{p} d \vec{w}+\bar{a}+t-\Phi_{0}\left(\mathcal{D}_{1}\right)\right)} \\
& \cdot \frac{\theta_{*}\left(\int_{p_{0}}^{p} d \vec{w}-J a\right) \theta_{*}(a-J z)}{\theta_{*}\left(\int_{p_{0}}^{p} d \vec{w}-J z\right) \theta_{*}(a-J a)} .
\end{aligned}
$$

Consider the function

$$
\rho_{z, \lambda}(p)=\theta\left(\int_{p_{0}}^{p} d \vec{w}+\bar{z}+t-\Phi_{0}\left(\mathcal{D}_{1}\right)-\frac{1}{2 \pi i} \int_{\partial D} \log (\phi-\lambda) d \vec{w}\right)
$$

on $X$ which is in the numerator in the definition of $f_{z}$. Since $J[\underline{z}]=-[\underline{z}]$ and $[\bar{t}]=[t],[t]-\Phi_{0}\left(\mathcal{D}_{1}\right)=-J[t]-\Phi_{0}\left(\mathcal{D}_{1}\right)=-\Phi_{0}\left(\mathcal{D}_{1}\right)-\Phi_{0}(J \mathcal{D})+\Phi_{0}(J a)-\left[\Delta_{0}\right]=$ $-\Phi_{0}(\mathcal{D})+\Phi_{0}(J a)-\left[\Delta_{0}\right]=-[t]+\Phi_{0}(J a)-\Phi_{0}(a)=-[t]-\left[\omega_{a}\right]$. Therefore, for $\lambda<\operatorname{essinf}(\phi)$,

$$
\left[t-\Phi_{0}\left(\mathcal{D}_{1}\right)-\frac{1}{2 \pi i} \int_{\partial D} \log (\phi-\lambda) d \vec{w}\right]
$$

is in $\mathbb{R}^{g} / \mathbb{Z}^{g}$. Since the theta function $\theta$ associated with the double of a planar region does not vanish on $\mathbb{R}^{g}$, for a fixed $z$ there is at most a countable set of values of $\lambda$ off the interval $[\operatorname{ess} \inf (\phi), \operatorname{ess} \sup (\phi)]$ where the function $\rho_{z, \lambda}$ vanishes at $J z$. Therefore, for a fixed $z$ there is at most a countable set of points $\lambda$ off the interval $[\operatorname{ess} \inf (\phi)$, ess $\sup (\phi)]$ having the property that the function $\rho_{z, \lambda}$ defined on $X$ vanishes identically. If $f_{z}$ is not identically zero, then $f_{z}$ belongs to the character $\chi\left(a_{j}\right)=1 ; \chi\left(b_{j}\right)=\exp \left(\int_{\partial D} \log (\phi-\lambda) d w_{j}\right), j=1, \ldots, g$, and the divisor of $f_{z}$ has the form

$$
\left(f_{z}\right)=\mathcal{D}_{2}-J z+J a-\mathcal{D},
$$

where $\mathcal{D}_{2} \geq 0$ is of degree $g$ satisfying

$$
\Phi_{0}\left(\mathcal{D}_{2}\right)+\left[\Delta_{0}\right]=\Phi_{0}(J z)+\left[\Phi_{0}\left(\mathcal{D}_{1}\right)-t+\frac{1}{2 \pi i} \int_{\partial D} \log (\phi-\lambda) d \vec{w}\right] .
$$


Note that

$$
\begin{aligned}
{\left[\int_{p_{0}}^{p} d \vec{w}-\int_{p_{0}}^{J a} d \vec{w}+t-\Phi_{0}\left(\mathcal{D}_{1}\right)\right]=\left[\int_{p_{0}}^{p} d \vec{w}-\int_{p_{0}}^{J a} d \vec{w}\right]-\Phi_{0}\left(\mathcal{D}_{1}\right)-J[t] } \\
\quad=\left[\int_{p_{0}}^{p} d \vec{w}-\int_{p_{0}}^{J a} d \vec{w}\right]-\Phi_{0}\left(\mathcal{D}_{1}\right)-\Phi_{0}(J \mathcal{D})+\Phi_{0}(J a)-\left[\Delta_{0}\right] \\
\quad=\left[\int_{p_{0}}^{p} d \vec{w}\right]-\left(\Phi_{0}(\mathcal{D})+\left[\Delta_{0}\right]\right) .
\end{aligned}
$$

Therefore, for $p \in \mathcal{D}$,

$$
\left[\int_{p_{0}}^{p} d \vec{w}-\int_{p_{0}}^{J a} d \vec{w}+t-\Phi_{0}\left(\mathcal{D}_{1}\right)\right]=-\left(\Phi_{0}\left(\mathcal{D}^{\prime}\right)+\left[\Delta_{0}\right]\right)
$$

where $\mathcal{D}^{\prime} \geq 0$ and $\operatorname{deg} \mathcal{D}^{\prime}=g-1$. Now suppose that for a fixed non-real $\lambda$ and a fixed $z$ the function $f_{z}$ is not identically zero, and let $h_{z}=f_{z} H$. Then $h_{z}$ is a single-valued meromorphic function on $X \backslash \partial D$. The divisor of $h_{z}$ is the same as the divisor of $f_{z}$ and hence $h_{z}$ restricted to $\bar{D}$ is in $H_{\mathcal{D}}^{2}(d m)$. Moreover,

$$
(\phi-\lambda) h_{z}^{+}=h_{z}^{-} \quad \text { a.e. } m \text { on } \partial D .
$$

The restriction of $h_{z}$ to $J \bar{D}$ would be in $K_{\mathcal{D}}^{2, a}(d m) \subseteq K_{\mathcal{D}}^{2}(d m)$ except for the pole at $J z$ which must be removed. This can be removed by subtracting an appropriate multiple of $k_{z}^{\mathcal{D}}$. Recall that the reproducing kernel $k_{z}^{\mathcal{D}}$ of $H_{\mathcal{D}}^{2}(d m)$ is a meromorphic function given by

$$
\begin{aligned}
k_{z}^{\mathcal{D}}(\zeta) & =k(\zeta, z: \mathcal{D}) \\
& =\frac{\theta(\zeta+\bar{z}-a-\bar{a}-t) \theta(t) \theta_{*}(\zeta-J a) \theta_{*}(a-J z)}{\theta(\zeta-a-t) \theta(\bar{z}-\bar{a}-t) \theta_{*}(\zeta-J z) \theta_{*}(a-J a)}, \quad \zeta \in X_{0},
\end{aligned}
$$

with the divisor of the form $\left(k_{z}^{\mathcal{D}}\right)=\mathcal{D}_{z}-\mathcal{D}+J a-J z$, where $\mathcal{D}_{z} \geq 0$ is of degree $g$. Since $h_{z}$ and $k_{z}^{\mathcal{D}}$ have the same factor

$$
\frac{\theta_{*}\left(\int_{p_{0}}^{p} d \vec{w}-J a\right) \theta_{*}(a-J z)}{\theta_{*}\left(\int_{p_{0}}^{p} d \vec{w}-J z\right) \theta_{*}(a-J a)},
$$

for the constant $K(z)$ given by

$$
K(z)=\frac{\theta\left(t-\Phi_{0}\left(\mathcal{D}_{1}\right)-\frac{1}{2 \pi i} \int_{\partial D} \log (\phi-\lambda) d \vec{w}\right) \theta\left(\int_{J z}^{a} d \vec{w}+t\right) \theta(\bar{z}-\bar{a}-t)}{\theta\left(t-\bar{z}+\bar{a}-\Phi_{0}\left(\mathcal{D}_{1}\right)\right) \theta(t) \theta\left(\omega_{a}+t\right)} H(J z)
$$

and for the appropriate choice of $H(J z), h_{z}-K(z) k_{z}^{\mathcal{D}}$ a.e. $m$ on $\partial D$ is in $K_{\mathcal{D}}^{2, a}(d m)$ and $(\phi-\lambda) h_{z}^{+}=h_{z}^{-}$a.e. $m$ on $\partial D$. Let $g$ be the function defined on $X \backslash \partial D$ by $\left.g\right|_{D}=\left.h_{z}\right|_{D}$ and $\left.g\right|_{J D}=\left(h_{z}-K(z) k_{z}^{\mathcal{D}}\right) \mid J D$. Then,

$$
(\phi-\lambda) g^{+}=g^{-}+K(z) k_{z}^{\mathcal{D}} \quad \text { a.e. } m \text { on } \partial D .
$$

So $g$ is a solution to the inhomogeneous Hilbert barrier problem with the symbol $G=(\phi-\lambda)$ and $k=K(z) k_{z}^{\mathcal{D}}$. Applying the projection $P$ to both sides of the 
equation in $(*)$, we obtain $\left(T_{\phi}-\lambda\right) h_{z}=K(z) k_{z}^{\mathcal{D}}$. Therefore,

$$
\begin{aligned}
\left\langle\left( T_{\phi}\right.\right. & \left.-\lambda)^{-1} k_{z}^{\mathcal{D}}, k_{z}^{\mathcal{D}}\right\rangle \\
& =C(z) \frac{\theta\left(\omega_{z}+t-\Phi_{0}\left(\mathcal{D}_{1}\right)-\frac{1}{2 \pi i} \int_{\partial D} \log (\phi-\lambda) d \vec{w}\right)}{\theta\left(t-\Phi_{0}\left(\mathcal{D}_{1}\right)-\frac{1}{2 \pi i} \int_{\partial D} \log (\phi-\lambda) d \vec{w}\right)} \cdot \frac{H(z)}{H(J z)},
\end{aligned}
$$

where $C(z)=\frac{\theta\left(t-\bar{z}+\bar{a}-\Phi_{0}\left(\mathcal{D}_{1}\right)\right) \theta(t) \theta\left(\omega_{a}+t\right) \theta_{*}(a-J z) \theta_{*}(z-J a)}{\theta\left(\int_{J z}^{a} d \vec{w}+t\right) \theta\left(\int_{J a}^{z} d \vec{w}+t-\Phi_{0}\left(\mathcal{D}_{1}\right)\right) \theta_{*}(a-J a) \theta_{*}(z-J z)}$ is a constant depending only on $\mathcal{D}$ and $z$. Since the multiplicative Cauchy kernel $A(t, p) d t$ based at $b$ is the differential $\Omega_{p-b}$, if we choose $b=J z$, then $H(J z)=1$ and

$$
H(z)=\exp \left(-\int_{\partial D} \log (\phi-\lambda) d m_{z}\right)
$$

Thus, we have

$$
\begin{aligned}
\left\langle\left( T_{\phi}\right.\right. & \left.-\lambda)^{-1} k_{z}^{\mathcal{D}}, k_{z}^{\mathcal{D}}\right\rangle \\
& =C(z) \frac{\theta\left(\omega_{z}+t-\Phi_{0}\left(\mathcal{D}_{1}\right)-\frac{1}{2 \pi i} \int_{\partial D} \log (\phi-\lambda) d \vec{w}\right)}{\theta\left(t-\Phi_{0}\left(\mathcal{D}_{1}\right)-\frac{1}{2 \pi i} \int_{\partial D} \log (\phi-\lambda) d \vec{w}\right)} e^{-\int_{\partial D} \log (\phi-\lambda) d m_{z}},
\end{aligned}
$$

where $C(z)$ is the above constant. Since $\Psi_{k_{z}}(\lambda)=\left\langle\left(T_{\phi}-\lambda\right)^{-1} k_{z}^{\mathcal{D}}, k_{z}^{\mathcal{D}}\right\rangle$ is analytic off the spectrum of $T_{\phi}$, by analytic continuation of both sides of the last equation, one can extend the above equation for the case where $\lambda$ is an arbitrary non-real number. Also, for a given $\phi$ in $L^{\infty}(d m)$ there exists a sequence $\phi_{n}$ of smooth functions which converges pointwise boundedly to $\phi$. This fact and a further analysis can be used to remove the smoothness condition on the symbol $\phi$ from the above argument. The proof of the theorem now is complete.

\section{Spectral Character Results}

One can prove the following results by making minor modifications in the proofs of Corollaries of Clancey [5]. The modifications in the proofs are basically the replacements of the constant $t_{a}$ from the resolvent formula in [5] by the constant $t$ which appears in the above generalization of the resolvent formula.

Corollary 1. For a given representing measure $m$ and a given element $\phi$ in $L^{\infty}(d m), \bar{\phi}=\phi$, let $T_{\phi}$ be the associated Toeplitz operator on $H_{\mathcal{D}}^{2}(d m)$. For fixed $z$ in $D \backslash \operatorname{supp} \mathcal{D}$, let $k_{z}^{\mathcal{D}}$ denote the reproducing kernel in $H_{\mathcal{D}}^{2}(d m)$. If $T_{\phi}=\int x d E_{x}$ is the spectral resolution of $T_{\phi}$, then for almost all $x$ in $\mathbb{R}$

$$
\begin{gathered}
\frac{d\left\|E_{x} k_{z}^{\mathcal{D}}\right\|^{2}}{d x}=\lim _{y \downarrow 0}\left(\frac{1}{2 \pi i}\left\langle\left(T_{\phi}-(x+i y)\right)^{-1} k_{k}^{\mathcal{D}}, k_{z}^{\mathcal{D}}\right\rangle-\left\langle\left(T_{\phi}-(x-i y)\right)^{-1} k_{z}^{\mathcal{D}}, k_{z}^{\mathcal{D}}\right\rangle\right) \\
=\frac{1}{\pi} C(z) \operatorname{Im}\left(\Psi(x) e^{-i \pi \int_{A_{x}} d m_{z}}\right) e^{-\int_{\partial D} \log |\phi-x| d m_{z}} .
\end{gathered}
$$

Here,

$$
\psi(x)=\frac{\theta\left(\omega_{z}+t-\Phi_{0}\left(\mathcal{D}_{1}\right)+\frac{1}{2 \pi} \int_{\partial D} \log |\phi-x| d \vec{\nu}+\frac{i}{2} \int_{A_{x}} d \vec{\nu}\right)}{\theta\left(t-\Phi_{0}\left(\mathcal{D}_{1}\right)+\frac{1}{2 \pi} \int_{\partial D} \log |\phi-x| d \vec{\nu}+\frac{i}{2} \int_{A_{x}} d \vec{\nu}\right)},
$$

where $A_{x}=\{p \in \partial D: \phi(p)<x\}$ and $d \vec{\nu}=-\frac{1}{2} * d \vec{\omega}$ is a vector signed measure on $\partial D, \omega=\left(\omega_{1}, \ldots, \omega_{g}\right)$ and $\omega_{j}=\omega_{j}(z)$ is the harmonic measure of $b_{j}$ based at $z$, $j=1, \ldots, g$. 
Corollary 2. For a given representing measure $m$ and a given $\phi$ in $L^{\infty}(d m), \bar{\phi}=$ $\phi$, if $\phi$ is either a simple or analytic function on $\partial D$, then the singular continuous component of $T_{\phi}$ is absent.

Corollary 3. Let $D$ be a doubly connected region and let $m$ be a given representing measure. If $\phi$ is in $L^{\infty}(d m), \bar{\phi}=\phi$, then the singular continuous component of $T_{\phi}$ is absent.

\section{ACKNOWLEDGMENT}

The results in this paper form a portion of the author's doctoral dissertation [1] under the supervision of Professor K. F. Clancey.

\section{REFERENCES}

[1] G. Akbari Estahbanati, Riemann surfaces and Toeplitz operators on multiply connected planar regions, Dissertation, University of Georgia, 1993.

[2] J. A. Ball and K. F. Clancey, Reproducing kernels for Hardy spaces on multiply connected domains.

[3] K. F. Clancey, Toeplitz operators on multiply connected domains and theta functions, Operator Theory: Adv. and Appl. 35 (1988), 311-355. MR 91f:47038

[4] Representing measures on multiply connected planar domains, Illinois J. Math., vol. 352 (1991), 286-311. MR 92e:46110

[5] - On the spectral character of Toeplitz operators on multiply connected domains, Trans. Amer. Math. Soc., vol. 3232 (1991), 897-910. MR 91f:47039

[6] W. F. Donoghue, Jr., On the perturbation of spectra, Comm. Pure Appl. Math., 18 (1965), 559-579. MR 32:8171

[7] J. D. Fay, Theta Functions on Riemann Surfaces, Lecture Notes in Mathematics No. 352, Springer-Verlag, New York, 1973. MR 49:569

[8] D. Mumford, Tata Lectures on Theta. I, II, Birkhäuser Verlag, Basel, 1983. MR 86b:14017, 85h:14026

[9] J. D. Pincus and J. Xia, Symmetric and self-adjoint Toeplitz operators on multiply connected planar domains, J. Funct. Anal. 59 (1984), 397-444. MR 87i:47038

[10] M. Rosenblum, A concrete spectral theory for self-adjoint Toeplitz operators, Amer. J. Math. 87 (1965), 709-718. MR 31:6127

[11] E. I. Zverovich, Boundary value problems in the theory of analytic functions in Hölder classes on Riemann surfaces, Russian Mathematical Surveys 26 (1971), 117-192. MR 53:13593

Department of Mathematics and Computer Science, North Georgia College, Dahlonega, Georgia 30597

E-mail address: akbari@nugget.ngc.peachnet.edu 